\title{
Additive anti-allergic effects of anti-interleukin-33 and anti-Siglec-F treatments in a murine model of allergic asthma
}

\author{
TAE YOUNG JANG, CHANG-SHIN PARK, MYUNG-SHIN JEON, MIN-JEONG HEO, \\ KWANGMIN NA, YOUNG HYO KIM \\ Inha University College of Medicine, Incheon, South Korea
}

\begin{abstract}
Background: Anti-interleukin-33 (anti-IL-33) and anti-Siglec-F antibodies have potent anti-allergic effects on murine allergic asthma and rhinitis and induce eosinophil apoptosis.

Objective: We aimed to determine whether post-sensitization with anti-IL-33/anti-Siglec-F treatments exhibited more potent effects compared to individual treatments in a murine allergic asthma model.

Material and methods: Twenty-five BALB/c mice were separated into five groups $(n=5)$ : Group A (control), Group B (ovalbumin [OVA] challenge), Group C (OVA + anti-IL-33), Group D $(O V A+$ anti-Siglec-F), and Group E $(O V A+$ anti-IL-33 + anti-Siglec-F). Serum total/OVA-specific IgE, bronchoalveolar lavage (BAL) inflammatory cells and cytokines (IL-4 and IL-5), histopathological lung properties, and airway hyperreactivity were compared.

Results: Ovalbumin challenge induced strong immune and inflammatory responses with $>6$-fold IgE level increases; 10- to 25-fold BAL eosinophil, neutrophil, and lymphocyte count increases; and $>1.5$-fold IL-4 and IL-5 level increases $(p<0.05)$. Whereas anti-IL-33 reduced neutrophil counts, anti-Siglec-F and anti-IL-33/anti-Siglec-F reduced both eosinophil and neutrophil counts $(p<0.05)$. Individual treatments reduced OVA-mediated bronchiolar infiltration by 50\% $(p<0.05)$. Ovalbumin challenge increased airway hyperreactivity by 4-fold (Group B; $2000.0 \pm 671.8 \%$ increase in Penh) compared to controls (Group A; $445.7 \pm 33.5 \%$ increase in Penh) $(p=0.016)$. The anti-IL-33 (Group C: $1579.4 \pm 973.6 \%$ increase in Penh) and anti-Siglec-F (Group D: $930.4 \pm 236.5 \%$ ) groups demonstrated significantly reduced hyperreactivity $(p=0.029)$. Anti-IL-33/anti-Siglec-F therapy showed synergism towards neutrophil counts, IL-5 concentrations, bronchial infiltration, and hyperreactivity $(p<0.05)$.

Conclusions: Combination treatment with anti-IL-33/anti-Siglec-F had more potent anti-allergic effects, reducing eosinophilic infiltration through their additive effects in a murine allergic asthma model.
\end{abstract}

Key words: cytokines, asthma, allergy, interleukin-33, Siglec-F.

(Centr Eur J Immunol 2014; 39 (4): 426-433)

\section{Introduction}

Asthma is a major health concern worldwide and is characterized by chronic inflammation of the lower airway. About half of all asthma cases are known to be associated with predominant eosinophilic infiltration, namely allergic asthma [1]. Eosinophils play a role in inducing bronchoconstriction, airway mucus hypersecretion, Th2 polarization, and airway remodelling [2, 3].

As current treatment regimens containing traditional medications have been unsuccessful in controlling allergic asthma, there is a growing interest in antibody therapies targeting cell surface receptors stimulating mast cell and eosinophil responses and survival [4]. Interleukin (IL)-33, a member of the IL-1 superfamily, is involved in a variety of allergic responses such as prolonged survival of eosinophils and mast cells, increases in histamine release from mast cells, and increases in several Th 2 cytokines such as IL-4, IL-5, and IL-13 [5, 6]. In a murine model of allergic asthma, it was proven that an IL-33 blockade decreased eosinophilic infiltration, airway hyperreactivity, and Th2 cytokines [5, 7-10]. The anti-allergic effect of anti-IL-33

Correspondence: Prof. Young Hyo Kim, Inha University College of Medicine, Incheon, South Korea, e-mail: inhaorl@inha.ac.kr 
antibody has been identified in a murine model of allergic asthma and rhinitis $[11,12]$.

Siglecs (sialic acid-binding immunoglobulin-like lectins) are cell surface receptors with extracellular and cytoplasmic domains [13]. When a sialic acid-containing ligand binds to the extracellular domain of a Siglec, the immunoreceptor tyrosine-based inhibitory motif (ITIM) in the cytoplasmic domain exerts its own function [13]. Whereas human eosinophils are known to selectively express Siglec-8, murine eosinophils have Siglec-F. Siglec-8 and Siglec-F are known as functional paralogs, since they both induce eosinophil apoptosis upon activation [14, 15]. Results of earlier studies, such as the increased apoptosis of human eosinophils after incubation with Siglec-8 and marked eosinophilia in Siglec-F knockout mice, imply that human Siglec-8 and murine Siglec-F have important roles in the development of allergic diseases [16, 17]. We investigated the therapeutic effect of anti-Siglec-F antibody in a murine model of allergic rhinitis through the induction of eosinophilic apoptosis [18]. A previous study demonstrated anti-allergic effects, such as a decreased number of nose-scratching events, less infiltration of eosinophils into the nasal mucosa, and a significant decrease of Th2 cytokines after an intraperitoneal injection of anti-Siglec-F antibody.

In previous studies of animal models, it was proven that anti-IL-33 and anti-Siglec-F antibodies had potent anti-allergic effects (they significantly reduced the number of nose-scratching behaviours, Th2 cytokines in bronchoalveolar lavage fluid, and demonstrated significantly less eosinophilic infiltration) through inhibition of several allergic mechanisms. As these antibodies all work on eosinophils to induce apoptosis, a combination regimen using these two antibodies concurrently could have a much more powerful anti-allergic effect. However, no study has examined the anti-allergic effect of such a combination treatment to date.

Therefore, we aimed to evaluate the effect of an anti-IL-33/anti-Siglec-F combination treatment in a murine model of allergic asthma compared to single treatments using each antibody by evaluating: (1) the serum total and ovalbumin (OVA)-specific IgE, (2) the numbers of differential inflammatory cells such as eosinophils, neutrophils, and lymphocytes in bronchoalveolar lavage (BAL) fluid, (3) the histopathologic properties of the lung tissue, (4) the degree of airway hyperreactivity, and (5) the titres of Th2 cytokines such as IL-4 and IL-5 in BAL fluid.

\section{Material and methods}

\section{Animals}

Twenty-five female BALB/c mice were purchased from Orient Bio (Seongnam, Korea). All mice were 8-10 weeks of age and free from murine-specific pathogens. They were raised in a controlled environment with regular $12 \mathrm{~h}$ light/dark cycles and unrestricted food and water.
Throughout the experimental period, all mice were provided OVA-free food. All mice used in this study were handled according to a protocol approved by the Institutional Animal Care and Use Committee of the Inha University (INHA 130404-201).

\section{Systemic sensitization and intranasal challenge}

A sensitization and intranasal challenge for the induction of allergic asthma was performed as per a previous protocol with a slight modification [11, 12, 19]. Under pathogen-free conditions, OVA (40 $\mu \mathrm{g} / \mathrm{kg}$, Sigma-Aldrich, St. Louis, MO, USA), diluted in sterile normal saline along with aluminium hydroxide gel (alum adjuvant, $40 \mathrm{mg} / \mathrm{kg}$ ), was injected by an intraperitoneal route on days 1, 5, 14, and 21. Following these 4 intraperitoneal injections, daily intranasal challenges were performed from day 22 through day 35 , with OVA diluted in sterile normal saline $(20 \mu \mathrm{l}$ of $25 \mathrm{mg} / \mathrm{ml}$ OVA per mouse).

The antibody treatments used anti-mouse IL-33 antibody (R\&D Systems, Minneapolis, MN, USA) and mouse anti-Siglec-F antibody (Monoclonal Rat IgG2A clone no. 238047; R\&D Systems, Minneapolis, MN, USA). In initial studies, an immunization protocol was designed to optimize antibody responses in animal models [11-13, 18]. We injected antibodies before each intraperitoneal sensitization and intranasal OVA challenge (a total of 18 injections). In the present study, we adopted a protocol that better reflects the clinical treatment of allergic asthmatic patients already sensitized to allergens. Intraperitoneal antibody injections were only performed before intranasal instillations, without blocking the systemic sensitization process. Anti-IL-33 antibody was injected $30 \mathrm{~min}$ before each intranasal OVA challenge (from day 22 through day 35, $3.6 \mu \mathrm{g} / \mathrm{mouse}$ each time). An anti-Siglec-F injection was performed $1 \mathrm{~h}$ before each intranasal challenge on days 22, 24, 26, 28, 32, 34, and 35 (10 $\mu \mathrm{g} /$ mouse each time) [13]. For the combination treatment, anti-IL-33 antibody ( $3.6 \mu \mathrm{g} /$ mouse, $30 \mathrm{~min}$ before each intranasal OVA challenge) and an anti-Siglec-F injection ( $1 \mathrm{~h}$ before each intranasal challenge on days $22,24,26$, $28,32,34$, and $35 ; 10 \mu \mathrm{g} /$ mouse) were both administered.

The 25 mice were separated into five groups. In Group A (control group, $n=5$ ), the mice were sensitized and challenged with normal saline only. Mice in Group B (OVA challenge group, $n=5$ ) received intraperitoneal and intranasal OVA challenges for the induction of allergic asthma. Group C (anti-IL-33 treatment group, $n=5$ ) and Group D (anti-Siglec-F group, $n=5$ ) received a therapeutic antibody injection (anti-IL-33 or anti-Siglec-F antibody respectively) before intranasal OVA challenge according to the dose and schedule. Finally, in Group E (combined treatment group, $n=5$ ), mice received both anti-IL-33 and anti-Siglec-F antibody treatments. 


\section{Serum and bronchoalveolar lavage fluid collection}

Twenty-four hours after the last intranasal OVA challenge, serum and BAL fluid were collected. We used an aortic puncture technique for collecting serum. Bronchoalveolar lavage fluid was harvested by intra-tracheal lavage with normal saline (approximately $4 \mathrm{ml}$ ) [12].

\section{Histopathology}

The lung tissues were fixed in a $10 \%$ formalin solution for 3 weeks. Then, they were embedded in paraffin using standard methods. Four- $\mu$ m-thick sections were stained with haematoxylin and eosin (H\&E) to detect cellular infiltration. The number of infiltrated cells around a single bronchiole was counted in 10 random high-power fields $(400 \times)$, by 2 impartial examiners who were totally unaware of the aims of this study.

\section{Measurement of airway hyperreactivity}

We evaluated airway hyperreactivity according to a previously described method $24 \mathrm{~h}$ after the last intranasal OVA challenge $[20,21]$. Mice were placed in a plethysmography chamber (All Medicus, Seoul, Korea) and baseline readings were acquired and averaged over $3 \mathrm{~min}$. Aerosolized methacholine $(0-50 \mathrm{mg} / \mathrm{ml})$ was nebulized for 3 min through the inlet of the main chamber. Readings were then taken and averaged over 3 min after each nebulization. Penh, determined as ((expiratory time)/(relaxation time -1$)) \times(($ peak expiratory flow $) /($ peak inspiratory flow)), according to the manufacturer's protocol, is used as a measure of airway hyperreactivity to methacholine. Results are expressed as the percentage increase after chal-

\section{A}

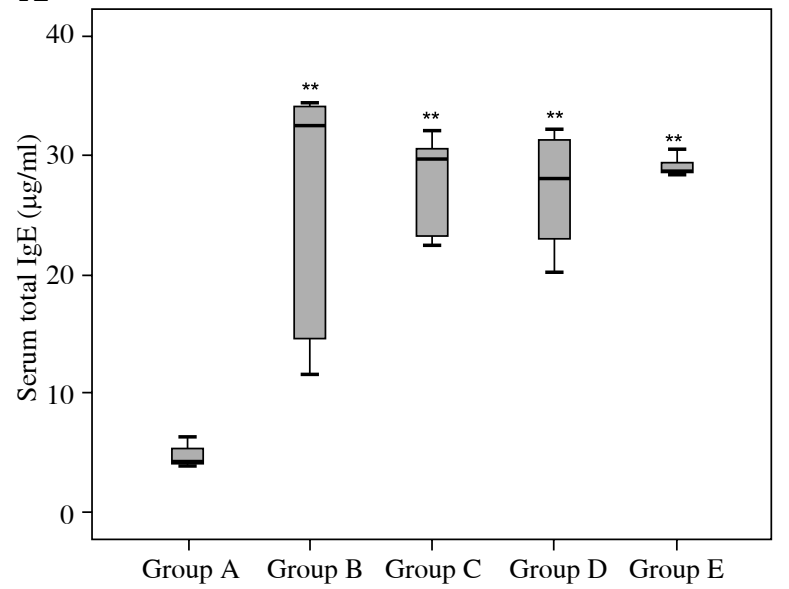

lenge for each concentration of methacholine (baseline Penh after saline challenge is expressed as 100\%).

\section{Enzyme-linked immunosorbent assays (ELISA)}

Serum titres of total and OVA-specific IgE were evaluated by ELISA according to previously described methods [22]. Total IgE was measured and compared with a mouse IgE standard (BD PharMingen, San Diego, CA, USA). We used optical density (OD) at $450 \mathrm{~nm}$ instead of calculating the concentration using a standard solution.

The levels of IL-4 and IL-5 were measured using individual ELISA kits (Biosource, Camarillo, CA, USA) as per the manufacturer's instructions and compared with known standards.

\section{Statistical analyses}

The data are expressed as the median and range. All statistical analyses were conducted with SPSS version 19.0 software (SPSS, Chicago, IL, USA). We used the Kruskal-Wallis test and Mann-Whitney $U$ test for comparisons of serum total and OVA-specific IgE levels, number of eosinophils, neutrophils, and lymphocytes in BAL fluid, and the number of inflammatory cells per bronchiole between the groups. $P$ values $<0.05$ were considered statistically significant.

\section{Results}

\section{Serum total and ovalbumin-specific immunoglobulin $\mathbf{E}$}

Ovalbumin challenge induced an approximately 6-fold increase in serum total IgE levels (Fig. 1A) and an approximately 10-fold increase in serum OVA-specific IgE levels (Fig. 1B) in Group B (OVA challenge) compared to Group A

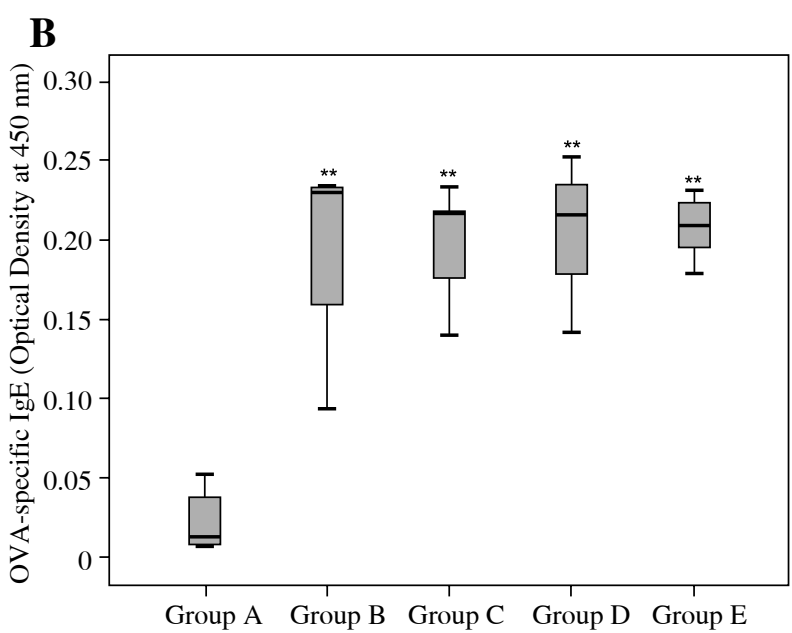

Fig. 1. Serum (A) total IgE and (B) OVA-specific IgE. Group A: control group, Group B: ovalbumin-induced allergic group, Group C: allergic group + treatment with anti-IL-33 antibody, Group D: allergic group + treatment with anti-Siglec-F antibody, and Group E: allergic group + combination treatment with both anti-IL-33 and anti-Siglec-F antibody (Kruskal-Wallis test and Mann-Whitney $U$ test; **significant difference with Group A, $p<0.01$ ) 

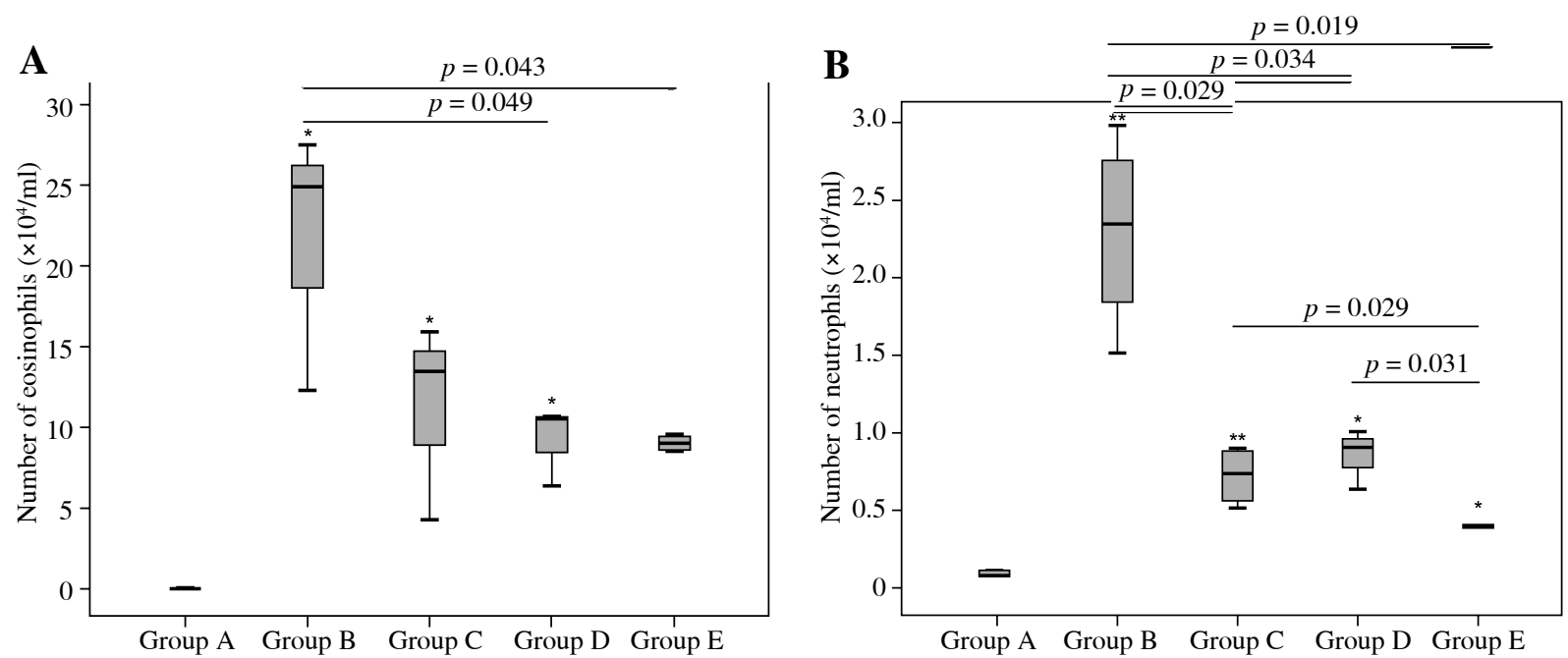

C

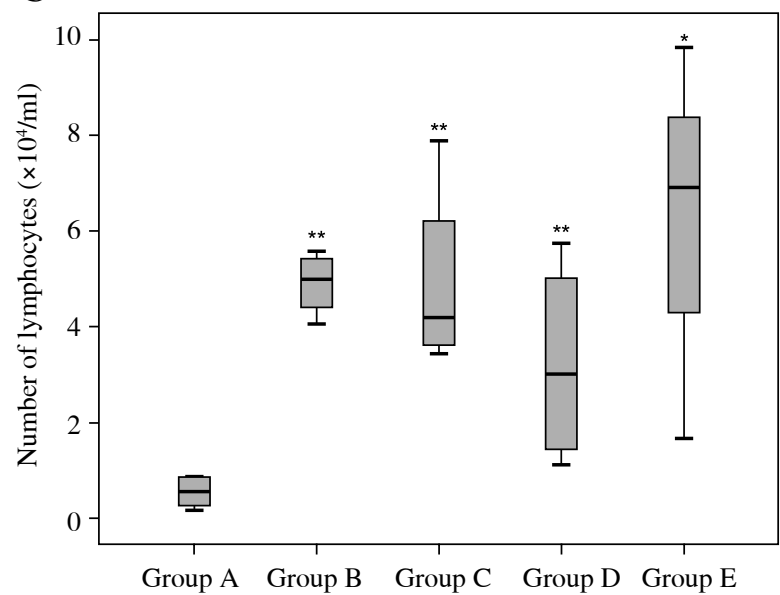

Fig. 2. Number of A) eosinophils, B) neutrophils, and C) lymphocytes in bronchoalveolar lavage fluid. Group A: control group, Group B: ovalbumin-induced allergic group, Group C: allergic group + treatment with anti-IL-33 antibody, Group D: allergic group + treatment with anti-Siglec-F antibody, and Group E: allergic group + combination treatment with both anti-IL-33 and anti-Siglec-F antibody (Kruskal-Wallis test and Mann-Whitney $U$ test; * $p<0.05$, **significant difference with Group A, $p<0.01$ )

(control) $(p<0.01)$. In contrast, none of the antibody treatments significantly reduced the total or OVA-specific IgE levels compared to Group B.

\section{Number of eosinophils, neutrophils, and lymphocytes in BAL fluid}

Ovalbumin challenge induced robust inflammatory and immune responses characterized by an approximately 25 -fold increase in eosinophil counts (Fig. 2A), an approximately 20-fold increase in neutrophil counts (Fig. 2B), and a 10-fold increase in lymphocyte counts (Fig. 2C) in BAL fluid (Group B vs. Group A; $p<0.05)$. The anti-IL-33 antibody treatment (Group C) reduced neutrophil counts, whereas anti-Siglec-F antibodies (Group D) reduced both eosinophil and neutrophil counts compared to Group B $(p<0.05)$. The combined treatment (Group E) only showed additivity towards neutrophil counts (Groups C and D, $p=0.029$ and 0.031, respectively).

\section{Histopathology}

A histological examination indicated that the bronchioles of OVA-challenged animals (Group B) showed significantly more inflammatory cell infiltration than those of control animals (Group A) (Fig. 3A). Both the single anti-IL-33 (Group C) and single anti-Siglec-F (Group D) antibody treatments significantly reduced cellular infiltration induced by OVA. Fur- 

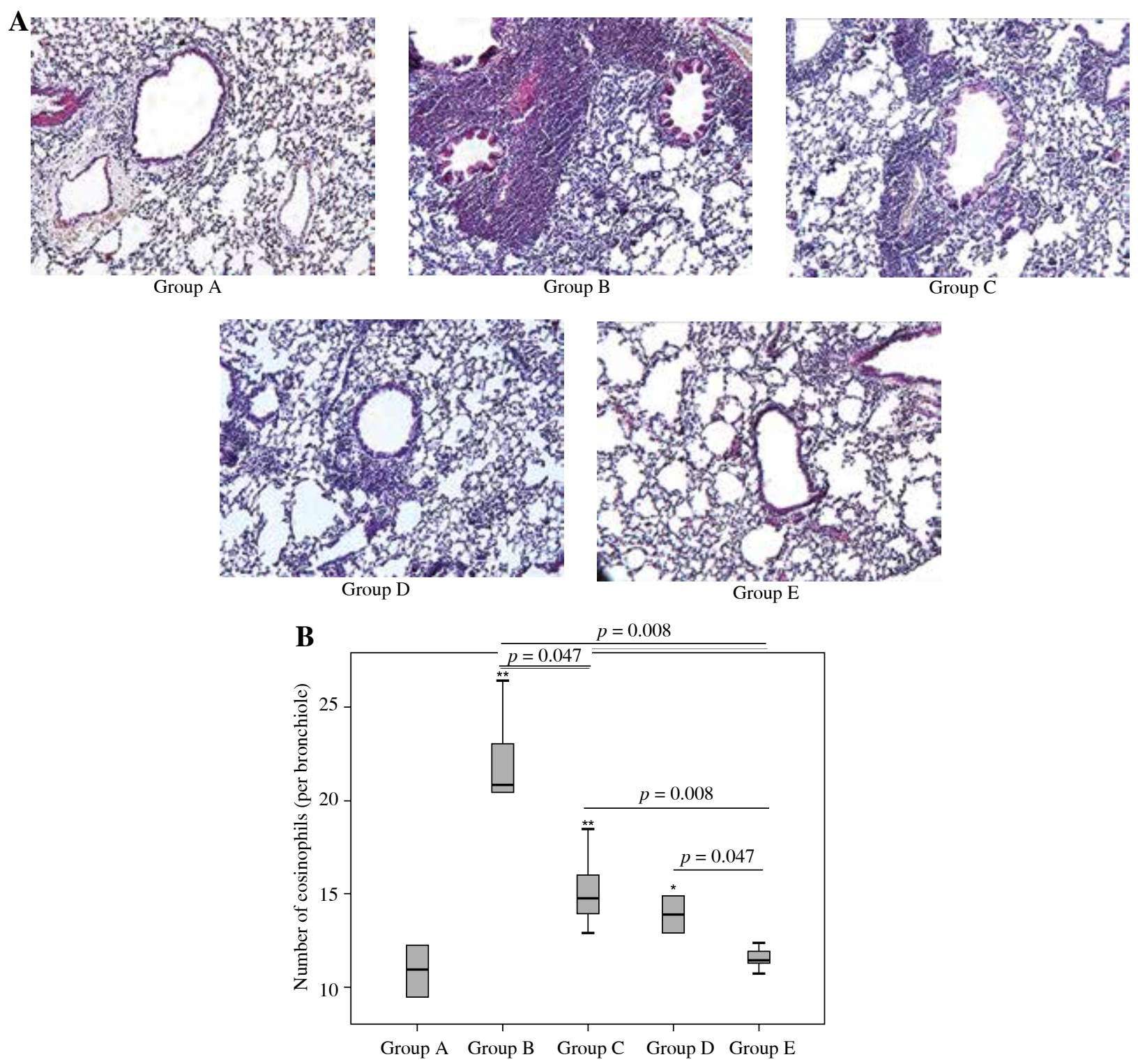

Fig. 3. A) A histopathologic examination of the lungs. Group A: control group, Group B: ovalbumin-induced allergic group, Group C: allergic group + treatment with anti-IL-33 antibody, Group D: allergic group + treatment with anti-Siglec-F antibody, and Group E: allergic group + combination treatment with both anti-IL-33 and anti-Siglec-F antibody (H\&E stain, 200×). B) Comparison of the number of eosinophils (per bronchiole) (Kruskal-Wallis test and Mann-Whitney $U$ test; ${ }^{*} p<0.05,{ }^{*}$ significant difference with Group A, $p<0.01$ )

thermore, anti-IL-33/anti-Siglec-F combination therapy (Group E) was more efficient than either single antibody treatment in reducing inflammatory cell infiltrations $(p<0.05)$. The impact of the various treatments on the number of eosinophils per bronchiole (Fig. 3B) was consistent with the data obtained from the BAL fluid (Fig. 2A). The single antibody treatments suppressed
OVA-mediated eosinophil infiltration by $\sim 50 \%$, whereas the anti-IL-33/anti-Siglec-F therapy returned eosinophil counts to the control levels demonstrated by Group A.

\section{Airway hyperreactivity}

Plethysmograph analysis showed that OVA challenge enhanced airway hyperreactivity to $50 \mathrm{mg} / \mathrm{ml}$ methacholine 
by 4 -fold (Group B; $2000.0 \pm 671.8 \%$ increase in Penh) compared to control values (Group A; $445.7 \pm 33.5 \%$ increase in Penh) (Fig. 4; $p=0.016$ ). A single antibody treatment with anti-IL-33 (Group C: $1579.4 \pm 973.6 \%$ increase in Penh) or anti-Siglec-F (Group D: $930.4 \pm 236.5 \%$ ) significantly reduced the effects of OVA $(p=0.029)$. In contrast, anti-IL-33/anti-Siglec-F treatment (Group E) restored normal hyperreactivity $(463.2 \pm 65.9 \%, p=0.029)$.

Cytokine analysis of the BAL fluid indicated that the OVA challenge raised IL-4 and IL-5 concentrations by 3-fold and 1.5-fold, respectively (Fig. 5). The antibody treatments did not affect their concentrations, except for the anti-IL-33/anti-Siglec-F combination, which reduced IL-5 concentrations to control levels.

\section{Discussion}

The development of antibody-based therapies is gaining popularity for the treatment of poorly controlled allergic asthma. The potential of anti-IL-33 and anti-Siglec-F antibodies targeting mast cells and eosinophils was previously demonstrated $[11,12,23]$. The present study shows that anti-IL-33/anti-Siglec-F combination therapy exerts a more potent effect on several aspects of allergic asthma, namely BAL neutrophils and IL-5, bronchial eosinophilic infiltrations, and airway hyperreactivity.

As anti-IL-33 and anti-Siglec-F both target eosinophils to enhance their apoptosis, we hypothesized that combination treatment using these antibodies could have a more potent anti-allergic effect. Compared to the single treatment groups (Groups $\mathrm{C}$ and $\mathrm{D}$ ), the combination

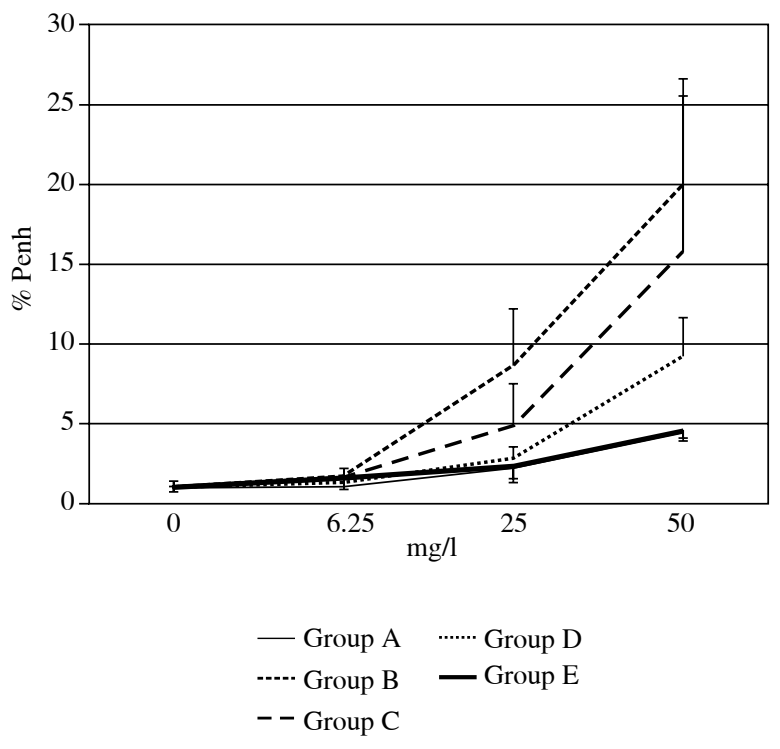

Fig. 4. Change in \% Penh with increasing concentrations of methacholine. Group A: control group, Group B: ovalbumin-induced allergic group, Group C: allergic group + + treatment with anti-IL-33 antibody, Group D: allergic group + treatment with anti-Siglec-F antibody, and Group E: allergic group + combination treatment with both anti-IL-33 and anti-Siglec-F antibody
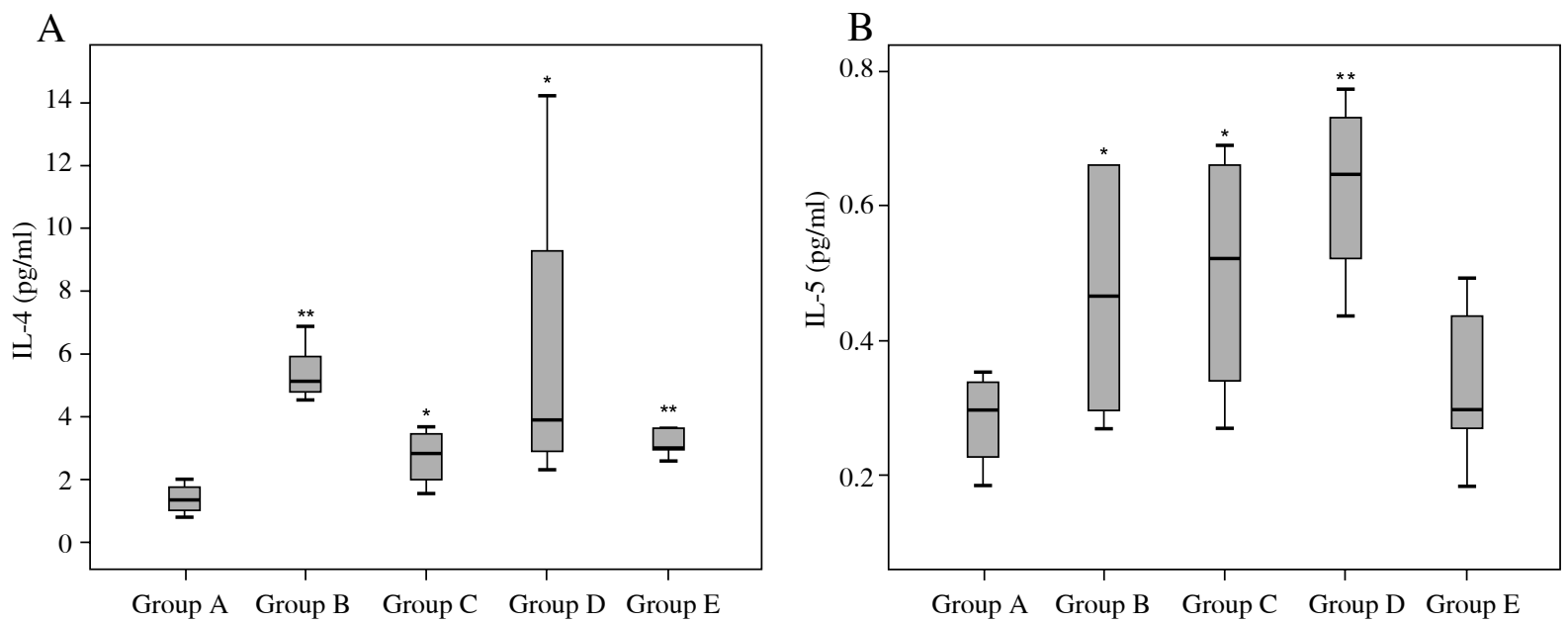

Fig. 5. The titres of A) IL-4 and B) IL-5 in bronchoalveolar lavage fluid. Group A: control group, Group B: ovalbumin-induced allergic group, Group C: allergic group + treatment with anti-IL-33 antibody, Group D: allergic group + treatment with anti-Siglec-F antibody, and Group E: allergic group + combination treatment with both anti-IL-33 and anti-Siglec-F antibody (Kruskal-Wallis test and Mann-Whitney $U$ test; $* p<0.05$, **significant difference with Group A, $p<0.01$ ) 
treatment using both antibodies induced a much greater decrease in inflammatory cell infiltration, especially that of eosinophils. Therefore, the mechanism of the more potent anti-allergic effect of the combination regimen is through its additive effects in inducing apoptosis of eosinophils. Cytokine analysis of the BAL fluid revealed that the titre of IL-5 was significantly more decreased to nearly normal levels after combination treatment, which was not the case for IL-4. The impact of combination therapy on IL-5 is consistent with the key role of this cytokine in the differentiation, activation, and survival of eosinophils. The IL-5-eosinophil axis is a major pathway in allergic inflammation [24]. In contrast, the IL-4/IL-13 pathway promotes mucus hypersecretion and airway remodelling [25].

The impact of antibodies on BAL neutrophil counts requires further mention, because it was quite unexpected. After combination treatment, Group E showed a greater decrease in neutrophils in BAL fluid compared to the single-treatment groups. This finding is an important subject because it raises issues about the specificity of anti-Siglec-F antibodies (in other words, whether anti-Siglec-F antibody could bind to another kind of Siglec receptor on the cellular membrane of neutrophils to induce its apoptosis). For example, Siglec-9 identified on neutrophils reduces neutrophil recruitment to the lungs [26]. More studies are needed to evaluate this possibility of cross-linking and could yield more relevant findings.

Evaluating the degree of airway hyperreactivity, Group C showed decreased Penh compared to Group B (although without statistical significance). In fact, intact IL-33 released from damaged bronchial epithelial cells plays an important role in the development of airway hyperreactivity by increasing IL-4 and IL-13 [27]. Group D showed a significant decrease in Penh compared to Group B. Through the combination regimen, Group E showed a much greater decrease in airway hyperreactivity compared to Group C and Group D, nearly reaching the values observed in Group A (control group). This is the first study to confirm the anti-allergic effect of combination treatment by proving both histopathologic and functional improvements.

Ideally, we should include a null treatment group, which uses irrelevant and isotype-matched antibody before each intranasal OVA instillation. Although the dosage and dosing schedule of both antibodies are not too high and have already been accepted as the relevant protocol in previous studies [11-13], we should keep in mind that therapeutic antibodies themselves could cause various unpredictable effects, ranging from protein delivery to antibody-specific effects such as immune response to a rat antibody to serum sickness. Furthermore, antibodies bound to a cell surface, such as anti-Siglec-F, can cause antibody-dependent cell-mediated cytotoxicity, complement-mediated cell lysis, and/or sequestration in spleen. And these many unpredictable effects could be another possible explanation for therapeutic effects of antibody treatment. To rule out these effects, more studies about the mechanism of these therapeutic antibodies should be performed in the nearby future.

Eosinophils play a protective role against some bacterial, viral, and parasitic infections [28-30]. The releases of several cytotoxic molecules such as eosinophilic cationic protein, major basic protein, and eosinophil-derived neurotoxin are important in providing antibacterial and anti-helminth immunity [31]. Therefore, one would worry about the increased chance of infection from a depletion of eosinophils. However, prior studies investigating anti-eosinophilic agents such as anti-IL-5 reported reasonable tolerance [32].

In conclusion, combination treatment with anti-IL-33/ anti-Siglec-F antibodies showed more potent anti-allergic effects and a greater decrease in airway hyperreactivity compared to single treatments through their additive effects in reducing eosinophilic infiltration in a murine model of allergic asthma.

The authors declare no conflict of interest.

This study was supported by the Basic Science Research Program through the National Research Foundation of Korea (NRF) funded by the Ministry of Education, Science, and Technology (NRF-2013R1A1A1006382), Bio \& Medical Technology Development Program (NRF2011-0019637), and received additional support from an Inha University Research Grant.

\section{References}

1. Woodruff PG, Modrek B, Choy DF, et al. (2009): T-helper type 2-driven inflammation defines major subphenotypes of asthma. Am J Respir Crit Care Med 180: 388-395.

2. Hogan SP, Rosenberg HF, Moqbel R, et al. (2008): Eosinophils: biological properties and role in health and disease. Clin Exp Allergy 38: 709-750.

3. Akuthota P, Wang HB, Spencer LA, Weller PF (2008): Immunoregulatory roles of eosinophils: a new look at a familiar cell. Clin Exp Allergy 38: 1254-1263.

4. Norman G, Faria R, Paton F, et al. (2013): Omalizumab for the treatment of severe persistent allergic asthma: a systematic review and economic evaluation. Health Technol Assess 17: 1-342.

5. Kurowska-Stolarska M, Kewin P, Murphy G, et al. (2008): IL-33 induces antigen-specific IL-5+ T cells and promotes allergic-induced airway inflammation independent of IL-4. J Immunol 181: 4780-4790.

6. Stolarski B, Kurowska-Stolarska M, Kewin P, et al. (2010): IL-33 exacerbates eosinophil-mediated airway inflammation. J Immunol 185: 3472-3480.

7. Kearley J, Buckland KF, Mathie SA, Lloyd CM (2009): Resolution of allergic inflammation and airway hyperreactivity is dependent upon disruption of the T1/ST2-IL-33 pathway. Am J Respir Crit Care Med 179: 772-781. 
8. Liu X, Li M, Wu Y, et al. (2009): Anti-IL-33 antibody treatment inhibits airway inflammation in a murine model of allergic asthma. Biochem Biophys Res Commun 386: 181-185.

9. Oshikawa K, Yanagisawa K, Tominaga S, Sugiyama Y (2002): Expression and function of the ST2 gene in a murine model of allergic airway inflammation. Clin Exp Allergy 32: 1520-1526.

10. Coyle AJ, Lloyd C, Tian J, et al (1999): Crucial role of the interleukin 1 receptor family member T1/ST2 in T helper cell type 2-mediated lung mucosal immune responses. J Exp Med 190: 895-902.

11. Kim YH, Yang TY, Park CS, et al. (2012): Anti-IL-33 antibody has a therapeutic effect in a murine model of allergic rhinitis. Allergy 67: 183-190.

12. Kim YH, Park CS, Lim DH, et al. (2012): Beneficial effect of anti-interleukin-33 on the murine model of allergic inflammation of the lower airway. J Asthma 49: 738-743.

13. Song DJ, Cho JY, Miller M, et al. (2009): Anti-Siglec-F antibody inhibits oral egg allergen induced intestinal eosinophilic inflammation in a mouse model. Clin Immunol 131: 157-169.

14. Floyd H, Ni J, Cornish AL, et al. (2000): Siglec-8. A novel eosinophil-specific member of the immunoglobulin superfamily. J Biol Chem 275: 861-866.

15. Tateno H, Crocker PR, Paulson JC (2005): Mouse Siglec-F and human Siglec-8 are functionally convergent paralogs that are selectively expressed on eosinophils and recognize 6'-sulfo-sialyl Lewis X as a preferred glycan ligand. Glycobiology 15: 1125-1135.

16. Nutku E, Aizawa H, Hudson SA, Bochner BS (2003): Ligation of Siglec-8: a selective mechanism for induction of human eosinophil apoptosis. Blood 101: 5014-5020.

17. Zhang M, Angata T, Cho JY, et al. (2007): Defining the in vivo function of Siglec-F, a CD33-related Siglec expressed on mouse eosinophils. Blood 109: 4280-4287.

18. Kim YH, Park CS, Lim DH, et al. (2013): Antiallergic effect of anti-Siglec-F through reduction of eosinophilic inflammation in murine allergic rhinitis. Am J Rhinol Allergy 27: 187-191.

19. Saito H, Matsumoto K, Denburg AE, et al. (2002): Pathogenesis of murine experimental allergic rhinitis: a study of local and systemic consequences of IL-5 deficiency. J Immunol 168: 3017-3023.

20. Kumar RK, Herbert C, Webb DC, et al. (2004): Effects of anticytokine therapy in a mouse model of chronic asthma. Am J Respir Crit Care Med 170: 1043-1048.

21. Foster PS, Ming Y, Matthei KI, et al. (2000): Dissociation of inflammatory and epithelial responses in a murine model of chronic asthma. Lab Invest 80: 655-662.

22. El Gazzar M, El Mezayen R, Marecki JC, et al. (2006): Anti-inflammatory effect of thymoquinone in a mouse model of allergic lung inflammation. Int Immunopharmacol 6: 11351142 .

23. Zimmermann N, McBride ML, Yamada Y, et al. (2008): Siglec-F antibody administration to mice selectively reduces blood and tissue eosinophils. Allergy 63: 1156-1163.

24. Stein ML, Munitz A (2010): Targeting interleukin (IL) 5 for asthma and hypereosinophilic diseases. Recent Pat Inflamm Allergy Drug Discov 4: 201-209.

25. Oh CK, Geba GP, Molfino N (2010): Investigational therapeutics targeting the IL-4/IL-13/STAT-6 pathway for the treatment of asthma. Eur Respir Rev 19: 46-54.

26. McMillan SJ, Sharma RS, McKenzie EJ, et al. (2013): Siglec-E is a negative regulator of acute pulmonary neutrophil inflammation and suppresses CD11b $\beta 2$-integrin-dependent signaling. Blood 121: 2084-2094.

27. Liew FY, Pitman NI, McInnes IB (2010): Disease-associated functions of IL-33: the new kid in the IL-1 family. Nat Rev Immunol 10: 103-110.

28. Lotfi R, Lee JJ, Lotze MT (2007): Eosinophilic granulocytes and damage-associated molecular pattern molecules (DAMPs): role in the inflammatory response within tumors. J Immunother 30: 16-28.

29. Kvarnhammar AM, Cardell LO (2012): Pattern-recognition receptors in human eosinophils. Immunology 136: 11-20.

30. Klion AD, Nutman TB (2004): The role of eosinophils in host defense against helminth parasites. J Allergy Clin Immunol 113: 30-37.

31. Shin MH, Lee YA, Min DY (2009): Eosinophil-mediated tissue inflammatory responses in helminth infection. Korean J Parasitol 47: S125-131.

32. Bochner BS, Gleich GJ (2010): What targeting eosinophils has taught us about their role in diseases. J Allergy Clin Immunol 126: 16-25. 